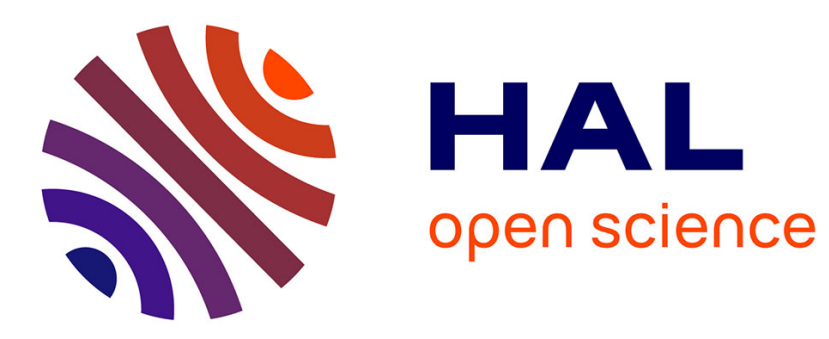

\title{
Pseudotime domain joint diving-reflected FWI using graph-space optimal transport
}

Giuseppe Provenzano, Wei Zhou, Romain Brossier, Ludovic Métivier

\section{To cite this version:}

Giuseppe Provenzano, Wei Zhou, Romain Brossier, Ludovic Métivier. Pseudotime domain joint diving-reflected FWI using graph-space optimal transport. First International Meeting for Applied Geoscience \& Energy, Sep 2021, Denver, United States. pp.797-801, 10.1190/segam2021-3583318.1 . hal-03404578

\section{HAL Id: hal-03404578 \\ https://hal.science/hal-03404578}

Submitted on 26 Oct 2021

HAL is a multi-disciplinary open access archive for the deposit and dissemination of scientific research documents, whether they are published or not. The documents may come from teaching and research institutions in France or abroad, or from public or private research centers.
L'archive ouverte pluridisciplinaire HAL, est destinée au dépôt et à la diffusion de documents scientifiques de niveau recherche, publiés ou non, émanant des établissements d'enseignement et de recherche français ou étrangers, des laboratoires publics ou privés. 


\section{Pseudotime domain joint diving-reflected FWI using graph-space optimal transport}

Giuseppe Provenzano*, Wei Zhou, Romain Brossier, ISTerre, Univ. Grenoble Alpes; Ludovic Métivier, LJK, CNRS, Univ. Grenoble Alpes

\section{SUMMARY}

Reflection waveform inversion (RWI) updates the the P-wave velocity $\left(V_{p}\right)$ macromodel beyond the depths sampled by diving waves, by exploiting wide scattering angle wavepaths in a reflective subsurface. Joint diving and reflection waveform inversion (JFWI) combines RWI and early-arrival waveform inversion (EWI), thereby constraining the shallow subsurface whilst enriching the low-wavenumber content of the deep $V_{p}$ model with reflections. In depth-domain $V_{p}$ inversion, ensuring consistency between reflectors positions and model kinematics comes at the cost of repeated least-square migrations, combined with carefully designed offset weighting. In order to efficiently address such co-dependency between reflective and kinematic parameters, we propose to cast JFWI in the pseudotime domain. As the velocity is updated, the reflectors are passively repositioned consistently with $V_{p}$, honoring the zerooffset two-way-time seismic invariant, and keeping the shortspread reflections in phase. By combining a pseudotime approach with a graph-space optimal transport (GSOT) objective function, we show that it's possible to reconstruct a complex velocity macromodel from short offset $2 \mathrm{D}$ reflection data containing surface-related multiples and ghosts, starting from a 1D initial guess; compared to a depth-domain inversion, the computing cost is reduced of one order of magnitude, associated with a significant saving in man-time, thanks to a simpler design of data weighting and inversion strategy.

\section{INTRODUCTION}

Depth-domain P-wave velocity $\left(V_{p}\right)$ reconstruction in models containing interfaces suffers from the cross-talk between $V_{p}$ and reflectors positions. Two-way traveltime velocity analysis, by keeping the zero-offset traveltime unchanged, is more robust against $V p$-depth ambiguities, but relies on a locally $1 \mathrm{D}$ approximation limiting its scope to areas without significant lateral variations. Migration velocity analysis (MVA) in the depth-domain is amenable to handling more complex subsurfaces, but requires iterative migration and residual-moveout minimization at the image point (e.g., Chauris and Noble, 2001). Ray-based depth-domain tomographic methods, such as stereotomography, update simultaneously the subsurface scatterers positions and $V_{p}$ (Tavakoli F. et al., 2017); in this context, it has been recently shown that enforcing the consistency between subsurface scatterers positions and $V_{p}$ is highly beneficial (Sambolian et al., 2019).

An alternative pseudotime (or vertical time) domain (Doherty and Claerbout, 1976; Snieder et al., 1989; Alkhalifah et al., 2001) has been proposed for full waveform inversion (FWI, Virieux and Operto, 2009), with the aim to combine the subwavelength resolution of FWI, with the robustness of data- domain methods (Plessix, 2013). This approach, as well as migration-based travetime FWI (MBTT, e.g., Chavent et al., 1994; Chavent, 2017), honors the zero-offset traveltime seismic invariant and, by keeping the short-offset waveforms in phase, reduces the non-linearity of $V_{p}$ waveform inversion (Plessix, 2013).

Reflection waveform inversion (RWI) relies on a reflective initial model to update the tomographic component of $V_{p}$ beyond the depths sampled by diving waves (e.g. Vigh et al., 2019; Yao et al., 2020). Since reflectivity is a function of $V_{p}$, depthdomain RWI requires iterative re-migration of the model discontinuties (Brossier et al., 2015) and offset selection to attenuate the conflict between fixed reflectivity and evolving kinematics. RWI has shown to benefit from a pseudotime formulation, in which $V_{p}$ is updated to fit the reflection moveout while reflectors are repositioned consistently in depth (Brossier et al., 2015; Kryvohuz et al., 2019). Joint FWI enriches the RWI sensitivity kernel with the constraints of diving waves in the shallow part (Zhou et al., 2015), and it's therefore expected to equally benefit from keeping the reflectivity fixed in the datadomain rather than in depth. JFWI, as well as FWI (Virieux and Operto, 2009), suffers from phase ambiguity when the predicted traveltimes differs from the observed of more than half a dominant period (Brossier et al., 2015; Zhou et al., 2015). Therefore, it benefits from the use of objective functions robust to cycle-skipping, such as cross-correlation time-shift (e.g., Brossier et al., 2015; Wang et al., 2019; Yao et al., 2020); Graph-space optimal transport (GSOT, Métivier et al., 2019) in particular has been shown to perform well when starting from poor initial models in combination with JFWI, without loss of resolution compared to a $L^{2}$-norm objective function (e.g., $\mathrm{Li}$ et al., 2019; Provenzano et al., 2020)

In this paper, we cast JFWI in the pseudotime domain and combine it with a GSOT objective function. A realistic synthetic short-offset reflection dataset containing free-surface effects is used to demonstrate that: 1) JFWI in the pseudotime is more robust than in depth-domain, and reconstructs a macro$V_{p}$ model suitable as starting model for FWI; 2 ) the pseudotime inversion strategy doesn't require offset selection, and dramatically reduces the need to iteratively re-migrate the reflectivity; 3) the advantages of pseudotime starting from a 1D initial model are maximised when using GSOT with respect to $L^{2}$ norm.

\section{METHODOLOGY}

\section{Joint FWI of diving and reflected waves}

JFWI (Zhou et al., 2015), jointly inverts early arrivals and reflected waves to obtain tomographic $V_{p}$ updates both in the shallow subsurface sampled by diving waves and the deeper parts covered by reflection wavepaths. This is combined with a velocity-impedance $\left(V_{p}-I_{p}\right)$ parametrization enhancing the scale separation between low and high wavenumbers of the 


\section{Pseudotime JFWI with graph-space OT}
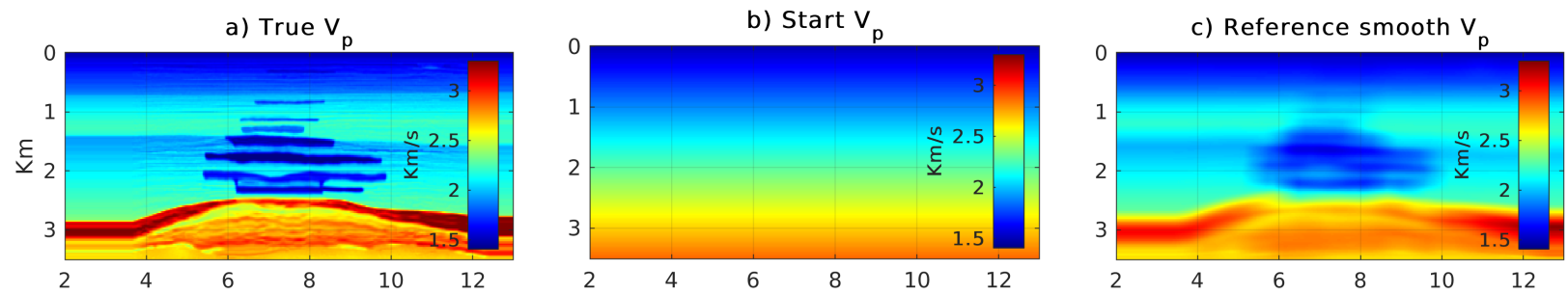

Figure 1: $V_{p}$ models: true and initial $V_{p}$ vs reference smooth $V_{p}$ (gaussian smoothing of true $V_{p}, L_{x}=0.6 \mathrm{Km}, L_{z}=0.3 \mathrm{Km}$ )

model. The objective function of JFWI is a weighted sum of early arrivals $(e)$ and reflections $(r)$ :

$$
\begin{aligned}
\chi\left[V_{p}\right]= & \digamma\left(W^{e}\left(d_{f}-d_{p}\left[V_{p}\right]\right)\right)+ \\
& \digamma\left(W^{r}\left(d_{f}-d_{p}\left[V_{p}, I_{p}\left(V_{p}\right)\right]\right)\right),
\end{aligned}
$$

where: $\digamma$ indicates a metric to measure the data distance; $W^{e}$ and $W^{r}$ are weights selecting early arrivals and reflections respectively; $d_{f}$ is the field data and $d_{p}$ is the data predicted in the current model. Note the dependency of $I_{p}$ on the current $V_{p}$ model in this depth domain formulation.

By neglecting high-order scattering effects (Zhou et al., 2015), the sensitivity kernel of Eq. 1 with respect to $V_{p}$ reads:

$$
\nabla \chi\left[V_{p}\right]=w_{e}^{2} u_{0} \star \lambda_{0}^{e}+u_{0} \star \delta \lambda^{r}+\delta u \star \lambda_{0}^{r}
$$

where: 1) the first term is the diving wave first Fresnel obtained by cross-correlation of the incident field $u_{0}$ and the background adjoint $\lambda_{0}^{e}$ generated by the diving wave residuals (EWI component weigthed by the scalar $\left.w_{e}\right) ; 2$ ) the second and third terms are the reflection rabbit ears resulting from the crosscorrelation of the incident wavefields $\left(u_{0}, \lambda_{0}^{r}\right)$ and the second order scattered wavefields $\left(\delta \lambda^{r}, \delta u\right)$ at the model discontinuities (RWI component).

\section{Pseudotime formulation}

In order to address the dependency of $I_{p}$ on $V_{p}$, and therefore the velocity-depth ambiguity, JFWI is re-formulated in the pseudotime domain, following Plessix (2013) and Brossier et al. (2015). The relationships between pseudotime $(\tau)$ and depth $(z)$ at each horizontal position are functions of $V_{p}$ :

$$
\tau(z)=\int_{0}^{z} d z^{\prime} / V_{p}^{z}\left(z^{\prime}\right) ; \quad z(\tau)=\int_{0}^{\tau} V_{p}^{t}\left(\tau^{\prime}\right) d \tau^{\prime}
$$

The depth domain $V_{p}^{z}, I_{p}^{z}$ model is mapped to the pseudotime domain $\tau$, discretized using $\Delta \tau=\Delta z / V_{p}(\max )$ and reaching $\tau_{\max }=z_{\max } / V_{p} \min$. The gradient (Eq.2) is computed in the depth domain, and reformulated in pseudotime using the derivation chain rule (Plessix, 2013):

$$
\nabla_{\tau(i)} \chi=\nabla_{z(i)} \chi-\int_{z_{i}}^{z_{\max }} \frac{d V_{p}^{d}}{d z} \frac{1}{V_{p}(z)} \nabla_{z} \chi d z
$$

After each $V_{p}$ update in the $\tau$ domain, the $V_{p}^{t}, I_{p}^{t}$ model is transformed to the modelling $(z)$ domain consistently with the new $V_{p}$; thereby, $I_{p}$ is fixed in pseudotime rather than in depth, and the near-zero offset waveforms are kept in phase by honoring the zero-offset seismic invariant.

\section{Graph-space optimal transport}

A graph-space OT (Métivier et al., 2019) objective function is used in order to reduce the liability to cycle-skipping at intermediate-to-long offsets. In GSOT, seismic traces are transformed from oscillatory signals into discrete distributions of $K$ unit-weight points in the 2D time-amplitude plane (the graphspace), so that OT can be applied with no loss of convexity. An optimal mapping $\sigma^{*}$ is found by minimising the distance between observed $\left(t(j), d_{f}(j)\right)$ and predicted $\left(t(i), d_{p}(i)\right)$ distributions, and the 2-Wasserstein based OT distance $h_{W^{2}}$ for each trace is computed as:

$$
h_{W^{2}}=\sum_{i=1}^{K}\left|t_{i}-t_{\sigma^{*}(i)}\right|^{2}+\frac{\Delta t}{A}\left|d_{i}^{p}-d_{\sigma^{*}(i)}^{f}\right|^{2}
$$

where the amplitude contribution is scaled by the ratio between the maximum expected time shift $\Delta t$ and the maximum amplitude $A$ in both observed and predicted data. Finally, the cost function is obtained as the summation of $h_{W 2}$ over sources and receivers, reweighted to honor trace-by-trace amplitude variations.

The GSOT adjoint source, obtained by $\delta h_{W^{2}} / \delta d_{p}$ (Plessix, 2006), is the data residuals computed between samples connected through the optimal assignment $\sigma^{*}$ (Métivier et al., 2019). In Eq.5, large $\Delta t$ values emphasize the convexity with respect to shifted patterns and therefore shall help to mitigate cycle skipping, whereas, as $\Delta t$ decreases, $\sigma^{*}$ tends to a local sample-by-sample mapping $\left(\sigma^{*}(i)=i\right)$, and $h_{W^{2}}$ approximates the behavior of the $L^{2}$ distance. The latter, can be therefore viewed as a special case of GSOT distance with $\Delta T=0$ in which the adjoint source is the data residual.

\section{EXAMPLE APPLICATION}

Synthetic marine reflection data have been generated on the Valhall 2D acoustic model ( $V_{p}$ and density), comprising a multilayered low-velocity zone (LVZ) and a deeper high velocity anticline (HVA). 128 shots are fired at $110 \mathrm{~m}$ spacing and the wavefield is recorded by a 229 channels surface streamer with group interval equal to $25 \mathrm{~m}$, and absolute offset ranging between 80 and $5700 \mathrm{~m}$. The source signature is a zero-phase Ricker with central frequency equal to $6.25 \mathrm{~Hz}$. Modeling is performed in the time domain with a 4 th order finite-difference scheme $(d x=25 m, d t=4 m s)$, PML absorbing boundaries and free-surface; therefore, the observed data contain surfacerelated multiples and ghosts. The starting model is 1D (Fig. 1), and it is made up by a water layer $\left(V_{p}=1500 \mathrm{~m}^{s-1}\right.$, density $=1$ 


\section{Pseudotime JFWI with graph-space OT}

$\mathrm{g} / \mathrm{cm}^{3}$ ) and a subsurface in which $V_{p}$ increases linearly with depth, while density is constant and equal to $2 \mathrm{~g} / \mathrm{cm}^{3}$, therefore the initial $I_{p}$ is simply a scaled version of $V_{p}$.

\section{$V_{p}$-macromodel building by JFWI}

Data windows are designed to separate early arrivals and reflected waves, in order to build the two components of the JFWI objective function in Eq.1; the limit between the two propagation regimes is simply given by the direct-wave arrival time. No scaling is applied to the the diving wave component of the adjoint source, i.e. $w^{e}=1$ (Eq. 2), since in reflection and diving residuals have the same order of magnitude in this freesurface example. The $V_{p}$-model building workflow begins with the inversion of the short-spread reflections (IpWI) in the starting one-dimensional $V_{p}$ (Fig.1b). The reconstructed $I_{p}$ is then used as the reflective component of the starting JFWI model. 20 iterations of depth preconditioned steepest descent are performed (Nocedal and Wright, 2006): the earliest 10 focus on reflections with zero-offset two-way time lower than $2.2 s$, after which the whole seismogram is included. This was necessary to allow for an accurate shallow subsurface imaging, which would have been otherwise neglected due to the high amplitude reflections at the LVZ. The choice of a steepest-descent optimization is justified by the need of a robust $V_{p}$ update in the presence of multiples and multi-scattering, in order to avoid overfitting, at the expense of convergence speed. In the GSOT case, the maximum expected time-shift $\Delta T$ (Eq. 5) is initially set to $0.3 \mathrm{~s}$ to privilege convexity with respect to traveltime differences, and later reduced to $0.1 \mathrm{~s}$ in the latest 10 iterations to approximate the resolution of the $L^{2}$ norm. Unlike in the depth-domain workflow outlined in Provenzano et al. (2020), here the pseudotime domain approach is tested with a more parsimonious inversion strategy, where no offset weighting is applied to the reflections, and model-building is performed in a single stage, i.e. without iteratively re-constructing $I_{p}$.

GSOT-based pseudotime JFWI achieves a reasonable $V_{p}$-macro -model reconstruction in a single stage, while depth-domain JFWI fails to converge towards the true model (Fig. 2a). Pseudotime GSOT yields a better prediction of the true $V_{p}$ trends than the $L^{2}$ equivalent (Fig. 2b-c), in particular in the shallow subsurface and at the bottom of the LVZ (Fig. 2d-f). The comparison between predicted and observed data (Fig.3) provides insights about the reason why pseudotime outperforms depth-domain JFWI: in panel a) the data predicted in the initial model are in phase at zero-offset, showing mismatches in reflection moveout and early-wave arrival times; after depthdomain JFWI (panel b), the data fit improves at long-to-intermediate offsets, at the expenses of the zero-offset waveform fit, because reflectivity is fixed in depth. On the contrary, in the pseudotime GSOT case, JFWI improves the longer-offsets waveform fit while keeping in phase the short-spread reflections (panel c), because in this formulation $I_{p}^{t}$ is independent of $V_{p}^{t}$; pseudotime $L^{2}$-norm inversion, on the other hand, though keeping the short offsets in phase, is cycle skipped at intermediateto-long offsets, particularly in the refracted arrivals (panel d).

\section{FWI after JFWI}

The pseudotime JFWI solutions obtained using GSOT and $L^{2}$
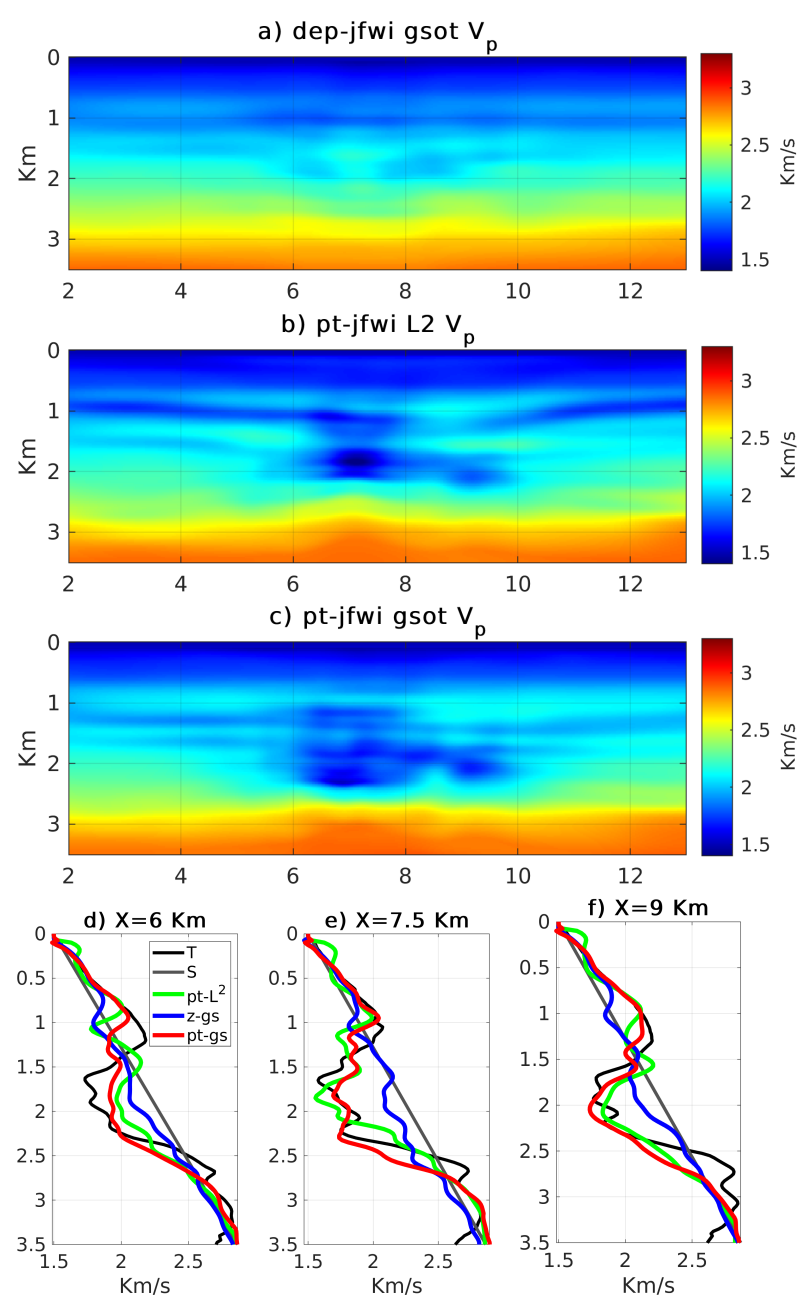

Figure 2: $V_{p}$-JFWI results: models and comparison with reference smooth $V_{p}$ (Fig.1c)

misfit functions are tested as starting models for $L^{2} V_{p}$-FWI, in conjunction with the uniform initial density model. The inversion is cast in a $V_{p}$-density parametrisation in which density is kept fixed; 30 iterations of 1-BFGS (Nocedal and Wright, 2006) are performed with linear depth preconditioning. The higher accuracy of GSOT-based pseudotime JFWI has a positive impact on the quality of the final FWI- $V_{p}$ model (Fig. $4 \mathrm{a}-\mathrm{b})$, in particular in the shallow subsurface $(<2 \mathrm{Km})$, and in placing the LVZ interfaces at the correct depths (Fig. 4c-e). The HVA image is reasonably retrieved, though the presence of surface-related multiples and the inaccurate starting density model prevent an accurate recovery of the $V_{p}$ absolute values.

\section{CONCLUSIONS}

Pseudotime domain JFWI obtains a complex macro $V_{p}$ model suitable for $V_{p}$-FWI, starting from a 1D initial guess and without requiring iterative re-migration of the reflective structures. This allowed for a reduction of the computing cost in the order of $1 / 10$ th compared to the depth-domain cycle workflow outlined in Provenzano et al. (2020); furthermore, unlike in the 


\section{Pseudotime JFWI with graph-space OT}
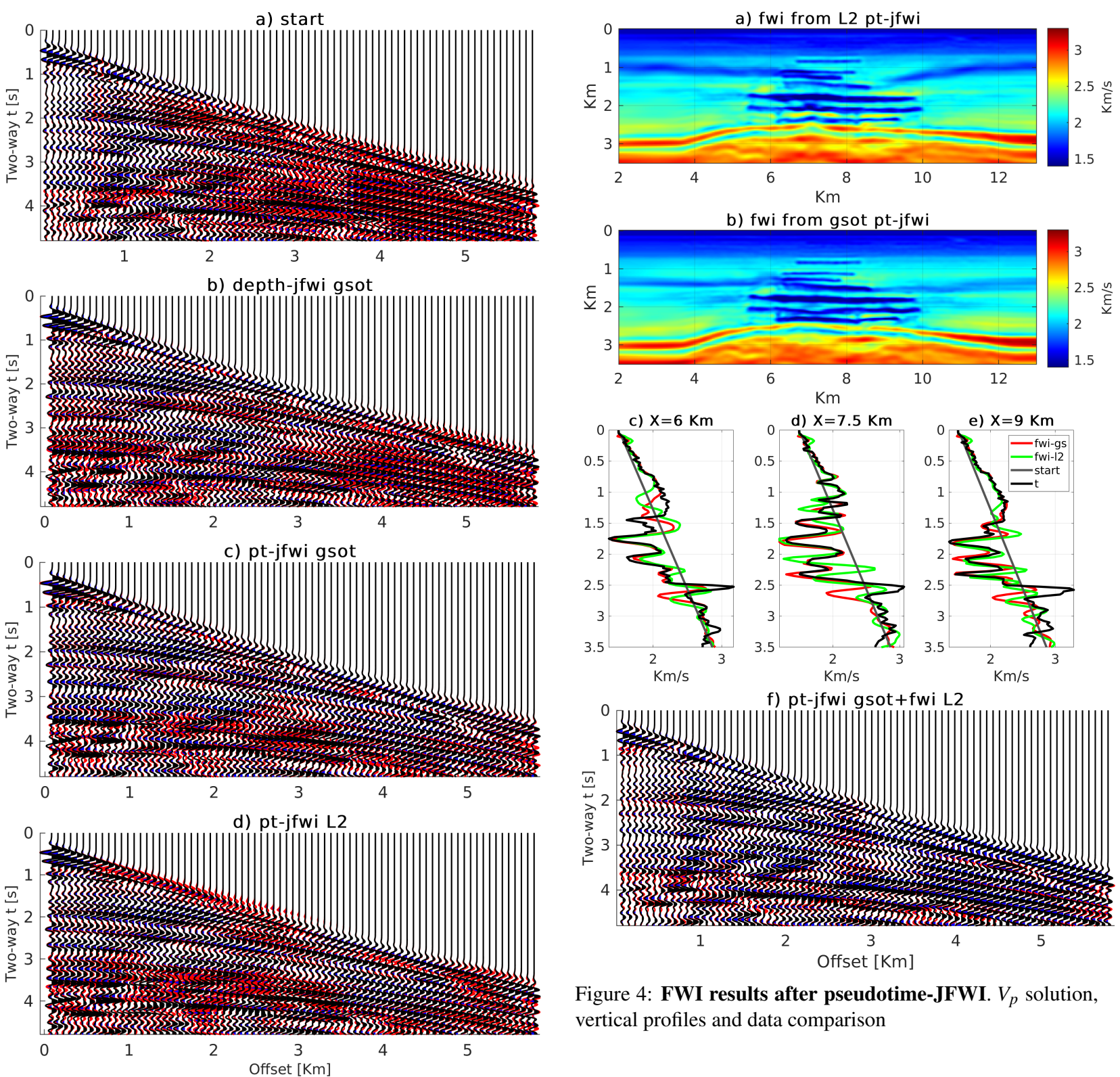

Figure 3: JFWI predicted vs observed data. Synthetics in red-blue, real data in black. Waveforms are in phase if black covers red.

latter study, the presence of surface-related multiples made the dataset particularly challenging for reflection-based $V_{p}$ inversion. The pseudotime-domain transform (Eq.4) has an extra cost in the order of $2 \%$; however, since no offset-weigthing is required, the inversion design is less user-dependent, which yields a significant pre-inversion time saving. FWI starting from the GSOT-based JFWI model achieves a higher accuracy than the one starting from $L^{2}$, thanks to a better reconstruction of the shallow $V_{p}$ driven by diving waves, cycle-skipped at long offsets in $L^{2}$; the same applies to the complex LVZ, where multi-scattering and internal multiples are important sources of phase ambiguity. In this dataset, GSOT has an extra-cost

Figure 4: FWI results after pseudotime-JFWI. $V_{p}$ solution, vertical profiles and data comparison

in the order of $20 \%$, though such a burden is expected to decrease proportionally in 3D applications and at higher frequencies (Métivier et al., 2019). Future work will assess the suitability of the methodology for more complex subsurfaces, in which steep dips could challenge the assumption of invariance of the zero-offset reflection traveltime and therefore partially hamper the advantages of the pseudotime formulation.

\section{ACKNOWLEDGEMENTS}

This study has been partially funded by the SEISCOPE consortium (https://seiscope2.osug.fr), sponsored by AKERBP, CGG, CHEVRON, EQUINOR, EXXON-MOBIL, JGI, SHELL, SINOPEC, SEISPROBE and TOTAL. This study was granted access to the HPC resources of CIMENT infrastructure (https://ciment. ujf-grenoble.fr) and CINES/ IDRIS/ TGCC under the allocation 046091 made by GENCI. 


\section{Pseudotime JFWI with graph-space OT}

\section{REFERENCES}

Alkhalifah, T., S. Fomel, and B. Biondi, 2001, The space-time domain: theory and modelling for anisotropic media: Geophysical Journal International, 144, 105-113.

Brossier, R., S. Operto, and J. Virieux, 2015, Velocity model building from seismic reflection data by full waveform inversion: Geophysical Prospecting, 63, no. 2, 354-367.

Chauris, H., and M. Noble, 2001, 2D velocity macro model estimation from seismic reflection data by local Differential Semblance Optimization: applications on synthetic and real data: Geophysical Journal International, 144, 14-26.

Chavent, G., 2017, Data space reflectivity and the migration based travel time approach to fwi: Presented at the 79th EAGE Conference and Exhibition 2017-Workshops.

Chavent, G., F. Clément, and S. Gòmez, 1994, Automatic determination of velocities via migration-based traveltime waveform inversion: A synthetic data example: SEG Technical Program Expanded Abstracts 1994, 1179-1182.

Doherty, S., and J. Claerbout, 1976, Structure independent velocity estimation: Geophysics, 41, 850-881.

Kryvohuz, M., H. Kuel, R. Plessix, and Y. Yang, 2019, Reflection full-waveform inversion with data-space lsrtm: Presented at the SEG International Exposition and 89th Annual Meeting.

Li, Y., R. Brossier, and L. Métivier, 2019, Joint FWI for imaging deep structures: A graph-space OT approach: SEG Technical Program Expanded Abstracts 2019, 1290-1294.

Métivier, L., R. Brossier, Q. Mérigot, and E. Oudet, 2019, A graph space optimal transport distance as a generalization of $L^{p}$ distances: application to a seismic imaging inverse problem: Inverse Problems, 35, 085001.

Nocedal, J., and S. J. Wright, 2006, Numerical optimization, 2nd ed.: Springer.

Plessix, R. E., 2006, A review of the adjoint-state method for computing the gradient of a functional with geophysical applications: Geophysical Journal International, 167, 495-503.

, 2013, A pseudo-time formulation for acoustic full waveform inversion: Geophysical Journal International, 192, 613-630.

Provenzano, G., R. Brossier, and L. Métivier, 2020, Joint FWI of diving and reflected waves using a graph space optimal transport distance: synthetic tests on limited-offset surface seismic data: Presented at the SEG Technical Program Expanded Abstracts 2020.

Sambolian, S., S. Operto, A. Ribodetti, B. Tavakoli, and J. Virieux, 2019, Parsimonious slope tomography based on eikonal solvers and the adjoint-state method: Geophysical Journal International, 218, 456-478.

Snieder, R., M. Y. Xie, A. Pica, and A. Tarantola, 1989, Retrieving both the impedance contrast and background velocity: a global strategy for the seismic reflection problem: Geophysics, 54, 991-1000.

Tavakoli F., B., S. Operto, A. Ribodetti, and J. Virieux, 2017, Slope tomography based on eikonal solvers and the adjoint-state method: Geophysical Journal International, 209(3), 1629-1647.

Vigh, D., X. Cheng, K. Jiao, and D. Sun, 2019, Keys to robust reflection-based full-waveform inversion: Presented at the 81st EAGE Conference \& Exhibition 2019.

Virieux, J., and S. Operto, 2009, An overview of full waveform inversion in exploration geophysics: Geophysics, 74, WCC1WCC26.

Wang, C., P. Farmer, D. Yingst, I. Jones, C. Calderon, and J. Brittan, 2019, Preconditioned reflection full waveform inversion for subsalt imaging: Presented at the 81st EAGE Conference \& Exhibition 2019.

Yao, G., D. Wu, and S. Wang, 2020, A review on reflection waveform inversion: Petroleum science, 17, 334-351.

Zhou, W., R. Brossier, S. Operto, and J. Virieux, 2015, Full waveform inversion of diving \& reflected waves for velocity model building with impedance inversion based on scale separation: Geophysical Journal International, 202, 1535-1554. 